\title{
AICAR Prevents Acute Olanzapine-Induced Disturbances in Glucose Homeostasis
}

\author{
Natasha D. Bush, Logan K. Townsend, and David C. Wright \\ Department of Human Health and Nutritional Sciences, University of Guelph, Guelph, Ontario, Canada \\ Received February 8, 2018; accepted March 22, 2018
}

\begin{abstract}
Olanzapine (OLZ) is an antipsychotic drug used in the treatment of schizophrenia. Although effective in reducing psychoses, OLZ causes acute increases in blood glucose. The acute effects of OLZ on hyperglycemia are likely caused by reductions in insulin secretion, insulin resistance, and increased hepatic glucose production. 5AMP-activated protein kinase (AMPK) is an energy sensor activated during exercise that can increase insulin sensitivity and insulin-independent glucose uptake in muscle. 5-Aminoimidazole-4-carboxamide ribonucleoside (AICAR) is a pharmacologic agent that, among other effects, can activate AMPK in vivo. Conversely, hypothalamic activation of AMPK has been suggested to mediate the hyperglycemic effects of OLZ. The purpose of this investigation was to determine whether
\end{abstract}

cotreatment with AICAR could prevent acute OLZ-induced hyperglycemia in lean and obese C57BL6/J mice. OLZ ( $5 \mathrm{mg} / \mathrm{kg}$, i.p.) caused rapid increases in blood glucose, a blunted insulin response, and pyruvate intolerance, all of which were prevented with AICAR cotreatment in both lean and obese mice. AICAR did not affect OLZ-induced changes in whole-body substrate oxidation or energy expenditure. Peripheral injection of AICAR, but not OLZ, activated AMPK signaling in the hypothalamus. The results of the current study provide evidence that AICAR prevents OLZ-induced hyperglycemia, despite increasing hypothalamic AMPK signaling. These protective effects were associated with the preservation of whole-body insulin action and reductions in markers of liver glucose production.

\section{Introduction}

Although second-generation antipsychotic (SGA) drugs have traditionally been used as a frontline treatment of schizophrenia, off-label use has increased dramatically over the past decade (Pringsheim and Gardner, 2014). SGAs have been increasingly prescribed for off-label conditions such as anxiety, attention deficit hyperactivity disorder, insomnia, and chemotherapyinduced nausea (Maher et al., 2011; Pringsheim and Gardner, 2014; Devlin and Panagiotopoulos, 2015; Bun et al., 2017; Chelkeba et al., 2017). SGAs, such as olanzapine (OLZ), have numerous metabolic side effects (Newcomer, 2005), including weight gain, dyslipidemia, hyperglycemia, insulin resistance, and an increased risk for the development of type 2 diabetes and cardiovascular disease (Bergman and Ader, 2005; Newcomer, 2005; Kessing et al., 2010; Lord et al., 2017; Reynolds and McGowan, 2017). Although impairments in glucose homeostasis

D.C.W. is a Tier II Canada Research Chair in Lipids Metabolism and Health. This work was supported by a Discovery Grant from the Natural Sciences and Engineering Research Council of Canada. N.D.B. was funded by a Canada Graduate Scholarship from the Canadian Institutes of Health Research and subsequently by an Ontario Graduate Scholarship. L.K.T. is funded by a scholarship from the Dairy Farmers of Ontario and a Doctoral Postgraduate Scholarship from the Natural Sciences and Engineering Research Council of Canada.

https://doi.org/10.1124/jpet.118.248393. often accompany weight gain itself, there is evidence that OLZ has direct diabetogenic effects independent of changes in body weight. Studies using both humans and rodents have shown increases in blood glucose within minutes to hours of OLZ treatment (Houseknecht et al., 2007; Chintoh et al., 2008, 2009; Boyda et al., 2010; Hahn et al., 2013; Ikegami et al., 2013). The acute effects of OLZ are exacerbated in conditions of preexisting obesity and impaired glucose homeostasis (Townsend et al., 2018), which is particularly troubling because individuals with schizophrenia are often obese and display perturbations in glucose homeostasis before taking antipsychotics (Subramaniam et al., 2014).

Although the mechanisms are still under investigation, OLZinduced hyperglycemia is thought to involve increases in hepatic glucose production (Chintoh et al., 2009; Ikegami et al., 2013), impaired insulin release (Boyda et al., 2010), systemic insulin resistance (Chintoh et al., 2008; Boyda et al., 2010), and an increased reliance on fat oxidation (Klingerman et al., 2014). In recent work, we have shown that the acute effects of OLZ are potentiated in mice with preexisting metabolic dysfunction, induced by 4 weeks of high-fat feeding (Townsend et al., 2018). This finding is important because those with schizophrenia often display perturbations in glucose homeostasis before treatment with SGAs (Bergman and Ader, 2005; van Nimwegen et al., 2008; Pillinger et al., 2017). Repeated, transient excursions in blood

ABBREVIATIONS: ACC, acetyl-CoA carboxylase; AICAR, 5-aminoimidazole-4-carboxamide ribonucleoside; AMPK, 5'AMP-activated protein kinase; ANOVA, analysis of variance; AUC, area under the curve; CLAMS, Comprehensive Laboratory Animal Monitoring System units; DMSO, dimethylsulfoxide; G6Pase, glucose 6 phosphatase; HFD, high-fat diet; ITT, insulin tolerance test; OLZ, olanzapine; PEPCK, phosphoenolpyruvate carboxykinase; RER, respiratory exchange ratio; SGA, second-generation antipsychotic; ZMP, 5-aminoimidazole-4-carboxamide ribonucleoside 5' monophosphate. 
glucose are harmful because they may lead to cardiovascular complications, including impaired endothelial function and increased oxidative stress (Ceriello et al., 2008); therefore, it is important to identify approaches to offset the acute metabolic side effects of these drugs. Unfortunately, many commonly prescribed glucose-lowering drugs (metformin, rosiglitazone, or glyburide) are not entirely effective in protecting against acute SGA-induced impairments in glucose homeostasis (Boyda et al., 2012).

One well known approach that can enhance glucose homeostasis is exercise (Richter et al., 1982; Holloszy, 2003; Richter and Hargreaves, 2013). Previous research in our laboratory has investigated the use of exercise to protect against OLZ-induced hyperglycemia (Castellani et al., 2018). Using C57BL6/J mice, it was found that exhaustive, but not moderate, exercise protected against OLZ-induced hyperglycemia. Unfortunately, exercise adherence in those taking SGAs is very poor, and thus it is unlikely that exercise would be easily incorporated into treatment regimens (Firth et al., 2015). Therefore, alternative mechanisms to combat the metabolic side effects of OLZ need to be explored.

Although not without controversy (Goodyear, 2008), the compound 5-aminoimidazole-4-carboxamide ribonucleoside (AICAR) has been suggested to act as an "exercise mimetic" (Narkar et al., 2008; Guerrieri and van Praag, 2015). AICAR is an adenosine analog that is phosphorylated and converted into 5-aminoimidazole-4-carboxamide ribonucleoside 5' monophosphate (ZMP) (Hasenour et al., 2014). Increases in ZMP, similar to those of AMP, activate the energy-sensing enzyme AMP-activated protein kinase (AMPK). The activation of AMPK in skeletal muscle improves insulin sensitivity (O’Neill et al., 2014; Kjøbsted et al., 2015), stimulates glucose uptake (Jørgensen et al., 2004), and enhances fat oxidation (O'Neill et al., 2014); AMPK activation in the liver leads to reductions in gluconeogenesis (Foretz et al., 2005). The effects of AICAR in the liver are likely not solely attributable to AMPK as the AICAR-mediated inhibition of glucose production was intact in liver-specific AMPK knockout mice (Hasenour et al., 2014).

In contrast to the peripheral effects of AICAR, this compound could conceivably potentiate the acute effects of SGAs on blood glucose. For example, intracerebroventricular administration of either OLZ or AICAR have both been shown to activate AMPK in the hypothalamus, and this was associated with increases in hepatic glucose production (Ikegami et al., 2013). This result raises concern regarding whether AICAR-dependent AMPK activation would be an effective approach to prevent OLZinduced hyperglycemia.

Within this context, the purpose of the present investigation was to determine whether AICAR could protect against OLZinduced increases in blood glucose. We hypothesized that AICAR cotreatment would attenuate OLZ-induced hyperglycemia in both lean and obese mice and that this would be associated with a preservation of whole body insulin action and reductions in markers of liver glucose output.

\section{Materials and Methods}

\section{Materials}

Olanzapine (2-methyl-4-(4-methyl-1-piperazinyl)-10H-thieno[2,3-b][1,5]benzodiazepine) (cat. no. O253750) and AICAR (5-aminoimidazole-4carboxamide ribonucleoside) (cat. no. A611700) were purchased from Toronto Research Chemicals (Toronto, ON, Canada). Dimethylsulfoxide
(DMSO) was from Wako Pure Chemical Industries (cat. no. 67-68-5; Richmond, VA). Blood glucose test strips and a Freestyle Lite handheld glucometer were acquired from Abbott Diabetes Care Inc. (Alameda, CA). Primary antibodies against phosphorylated AMPK $\alpha$ (T172) (cat. no. 2535), AMPK $\alpha$ (cat. no. 2532), phosphorylated acetyl-CoA carboxylase (ACC; S79; cat. no. 3661), and ACC (cat. no. 3676) were purchased from Cell Signaling (Danvers, MA). phosphoenolpyruvate carboxykinase (PEPCK; cat. no. 10004943) and glucose 6 phosphatase (G6Pase; cat. no. B1512) primary antibodies were purchased from Cayman Chemical Company (Ann Arbor, MI) and Santa Cruz Biotechnology (Dallas, TX), respectively. Vinculin primary antibody was purchased from Millipore (Billerica, MA). Secondary antibodies (donkey anti-rabbit and goat antimouse IgG) were purchased from Jackson ImmunoResearch (West Grove, PA). Insulin was purchased from Eli Lilly (Toronto, ON, Canada). Reagents for SDS-PAGE, including molecular weight marker, nitrocellulose membranes, and enhanced chemiluminescence, were purchased from Bio-Rad (Mississauga, ON, Canada). All additional substances, including those used to homogenize samples, were purchased from Sigma-Aldrich (St. Louis, MO).

\section{Animals}

Male C57BL6/J mice (8 weeks old) were obtained from Jackson Laboratory (Bar Harbor, ME). Mice were housed in individual cages on a 12-hour light/dark cycle (all experiments were performed during the light cycle) at $23^{\circ} \mathrm{C}$ with ad libitum access to water and standard rodent chow (7004-Teklad S-2335 Mouse Breeder Sterilizable Diet; Teklad Diets, Harlan Laboratories, Madison WI). After $~ 1$ week of acclimation, a separate cohort of mice were given a high-fat diet (HFD; $60 \%$ kcal from fat; Research Diets D12492) for 4 weeks. Food was removed 1 to 2 hours before and during all experiments except indirect calorimetry. Protocols were approved by the University of Guelph Animal Care Committee and followed the guidelines of the Canadian Council on Animal Care.

\section{Drug Preparation}

OLZ powder was first dissolved in DMSO at $10 \mathrm{mg} / \mathrm{ml}$ and subsequently added to a solution of sterile saline $(0.9 \% \mathrm{NaCl})$ and Kolliphor to make final concentrations $90 \%$ saline, 5\% Kolliphor, and 5\% DMSO/OLZ solution, making the final OLZ concentration $0.5 \mathrm{mg} / \mathrm{ml}$. The vehicle solution (vehicle 1) consisted of matched quantities of the three reagents but with no OLZ added. AICAR powder was dissolved in sterile saline $(0.9 \% \mathrm{NaCl})$ at a concentration of $25 \mathrm{mg} / \mathrm{ml}$. Sterile saline alone was used as the vehicle (vehicle 2) solution.

\section{Experimental Procedures}

AICAR Injections. Mice were injected with either AICAR $(250 \mathrm{mg} / \mathrm{kg}$, i.p.) or an equal amount of saline (vehicle 2). Mice were anesthetized 20 minutes after treatment with sodium pentobarbital (50 mg/kg, i.p.), after which liver and triceps muscle were collected and immediately frozen in liquid nitrogen before storage at $-80^{\circ} \mathrm{C}$.

Olanzapine and AICAR Tolerance Test. Mice were cotreated intraperitoneally with: 1 ) vehicle 1 and vehicle 2 ; 2) olanzapine $(5 \mathrm{mg} / \mathrm{kg})$ and vehicle $2 ; 3)$ vehicle 1 and AICAR $(250 \mathrm{mg} / \mathrm{kg}) ;$ or 4$)$ olanzapine ( $5 \mathrm{mg} / \mathrm{kg})$ and AICAR $(250 \mathrm{mg} / \mathrm{kg})$. This dose of olanzapine has been previously used to induce a significant rise in blood glucose (Ikegami et al., 2013). Additionally, this dose represents a dopamine $\mathrm{D}_{2}$ receptor occupancy of $\sim 85 \%-90 \%$ in rats, which, considering the significantly shorter half-life in rodents (2.5 hours in rodents vs. $21-54$ hours in humans), is roughly comparable to the clinically relevant occupancy of $60 \%-80 \%$ (Kapur et al., 2003). Blood glucose was measured from the tail vein $0,15,30,60$, and 90 minutes after drug treatment, and the glucose area under the curve (AUC) was calculated. Mice had access to water, but not food, during all tolerance tests.

Insulin Tolerance Test. Mice were treated with one of four drug cotreatments as described herein, followed by insulin injection 
(0.75 U/kg, i.p.) 30 minutes post-treatment. Blood glucose was measured at 0, 10 and 20 minutes post-insulin injection, and the glucose AUC was calculated. In the high-fat diet experiment, an additional blood glucose measurement was obtained 30 minutes after insulin injection.

Pyruvate Tolerance Test. Pyruvate ( $2 \mathrm{~g} / \mathrm{kg}$, i.p.) was injected 30 minutes after treatment with one of the four drug combinations (as already described ). Blood glucose was measured at 0, 15, 30, 60, and 90 minutes (HFD-fed mice only) after administration of pyruvate.

Hypothalamic AMPK and Gluconeogenic Protein Experiment. Mice were treated with one of the four drug combinations as previously described, and blood glucose was measured at 0 and 20 minutes post-injection to confirm that each treatment elicited the appropriate response (e.g., reduced blood glucose after AICAR administration). Mice were then anesthetized with sodium pentobarbital $(50 \mathrm{mg} / \mathrm{kg}$, i.p.) 20 minutes after treatment. The liver and hypothalamus were harvested and immediately frozen in liquid nitrogen before storage at $-80^{\circ} \mathrm{C}$. We chose this early time point to measure hypothalamic AMPK signaling as it coincides with the initial changes in blood glucose with treatment.

Western Blotting. Tissue samples were homogenized (FastPrep24; MP Biomedicals, Santa Ana, CA) in cell lysis buffer supplemented with phenylmethylsulfonyl fluoride and protease inhibitor cocktail [volume (microliters) using 40, 30, and 15 times weight (milligrams) of the hypothalamus, liver, and triceps, respectively]. Samples were then centrifuged at $1500 \mathrm{~g}\left(4^{\circ} \mathrm{C}\right)$ for 5 minutes (the hypothalamus was centrifuged at 10,000g for 15 minutes (MacPherson et al., 2015), the supernatant was collected, and a bicinchoninic acid assay was performed to determine protein concentration (Smith et al., 1985). Separation of equal amounts of protein was completed using $10 \%$ SDSPAGE gels, followed by a wet transfer onto nitrocellulose membrane (100 V for 60 minutes). Membranes were blocked for 1 hour using Trisbuffered saline/0.1\% Tween (TBST) with 5\% nonfat dry milk powder, followed by incubation with the appropriate primary antibody $(1: 1000$ dilution into TBST $/ 5 \%$ BSA) overnight at $4^{\circ} \mathrm{C}$. Membranes were incubated in the appropriate horseradish peroxidase-conjugated secondary antibody (1:5000 dilution into TBST/1\% nonfat dry milk powder) for 1 hour at room temperature. Protein content was detected using enhanced chemiluminescence followed by band quantification using densitometry (FluorChem HD Imaging System; Alpha Innotech, Santa Clara, CA). Protein content was expressed relative to an in-gel loading control (vinculin, GAPDH, or Ponceau) before expression relative to total protein levels (phosphorylated/total) if applicable.

Indirect Calorimetry. Mice were individually housed in Comprehensive Laboratory Animal Monitoring System units (CLAMS; Columbus Instruments, Columbus, $\mathrm{OH}$ ) at the beginning of their light (nonactive) phase. After an acclimation period of $\sim 24$ hours, mice were injected at 9:30 AM (just after the beginning of their light cycle) with one of four drug treatment groups as described previously and then immediately put back into the CLAMS for another 24 hours. Metabolic parameters, including $\mathrm{VO}_{2}$ (milligrams per kilogram per minute), $\mathrm{VCO}_{2}$ (milligrams per kilogram per minute), respiratory exchange ratio (RER; $\mathrm{VCO}_{2} / \mathrm{VO}_{2}$ ), heat production (kilocalories per minute), and activity levels (beam breaks) were measured over the next 24 hours.

\section{Statistical Analyses}

Data were analyzed using a two-tailed unpaired Student's $t$ test to compare AMPK activation in AICAR- and vehicle-treated mice. A twoway analysis of variance (ANOVA) with Tukey's post hoc analysis was used to examine the main effects and interactions of OLZ and AICAR treatment. When necessary, data were logarithmically transformed $\left(\log _{10}\right)$ to ensure equal variance and normal distribution. Data were expressed as mean \pm S.E., and statistical significance was set at $P<$ 0.05 . Given the magnitude of differences between groups in combination with low variability, our groups ( $n=4-8$ /group) provided sufficient power to detect differences. Statistical tests were completed using Sigma Plot version 11.0 (San Jose, CA), and figures were made using Graph Pad (Prism, version 7) (La Jolla, CA).

\section{Results}

AICAR Treatment Protects against OLZ-Induced Hyperglycemia. Numerous studies using cell culture and rodent models have shown that AICAR treatment increases the phosphorylation of AMPK and its downstream target, acetyl-CoA carboxylase (ACC) (Guigas et al., 2007; Gaidhu et al., 2009; Hasenour et al., 2014; O'Neill et al., 2014; Kjøbsted et al., 2015; Monaco et al., 2015). To confirm whether AICAR treatment leads to activation of AMPK in our hands, mice were injected with AICAR or an equivalent volume of saline, and the phosphorylation levels of AMPK and ACC were measured. In the liver, AICAR treatment resulted in a significant increase in the P-AMPK/AMPK $(P<0.01)$ and $(\mathrm{P})$-ACC/ACC $(P<0.05)$ ratios $(\sim 5$-fold and 2.5 -fold, respectively) (Fig. 1A). In triceps muscle, AICAR effected an increase of $\sim 3$-fold in P-ACC/ACC $(P<0.05)$ (Fig. 1B).

To examine whether AICAR can protect against OLZinduced acute hyperglycemia, mice were separated into four treatment groups and injected with OLZ, AICAR, or a combination of the two compounds. OLZ significantly increased blood glucose levels compared with vehicle-treated mice $(P<0.001)$, and this increase was completely prevented by cotreatment with AICAR $(P<0.001)$ (Fig. 1, D and $\mathrm{E}$ ).

AICAR Treatment Mitigates OLZ-Induced Reductions in the Insulin Response. To investigate how OLZ and AICAR might alter the systemic response to insulin, an insulin tolerance test (ITT) was performed after pretreatment with each of the four drug combinations. OLZ-treated mice demonstrated a blunted insulin response $(P<0.001)$ as shown by an increased glucose AUC after insulin treatment, and this response was alleviated with AICAR cotreatment $(P<0.001)$ (Fig. 2).

AICAR Inhibits Pyruvate-Induced Excursions in Blood Glucose after OLZ Treatment. To gain further insight into the prevention of OLZ-induced hyperglycemia using AICAR, a pyruvate tolerance test was performed after pretreatment with each of the four drug combinations. Pyruvate-induced increases in glucose levels were drastically potentiated with OLZ pretreatment $(P<0.001)$, and this effect was significantly blunted with AICAR $(P<0.001)$ (Fig. 3, A and C). To ensure that the AUC data were not solely influenced by differences in blood glucose levels immediately before pyruvate injection, blood glucose levels are also presented as percent change after pyruvate injection. From this standpoint, OLZ pretreatment still elicited a potentiated response to pyruvate $(P<0.05)$, which was significantly blunted with AICAR cotreatment $(P<0.001)$ (Fig. $3, \mathrm{~B}$ and D). Additionally, contrary to that observed with absolute values, a significant difference was evident between the AICAR pretreated group and the AICAR + OLZ pretreated group with respect to the relative values $(P<0.001)$.

It has been previously demonstrated that gluconeogenic enzyme mRNA levels, namely, glucose-6-phosphatase, are elevated with OLZ treatment (Ikegami et al., 2013). To elucidate whether changes in a marker of hepatic gluconeogenesis were correlated with changes in gluconeogenic enzyme protein content, liver G6Pase and PEPCK protein content were analyzed by Western blotting. No significant differences between treatment groups were found in the protein content of these enzymes (Fig. 3, E, F, and G). 
A Vehicle $\square$ AICAR

Liver

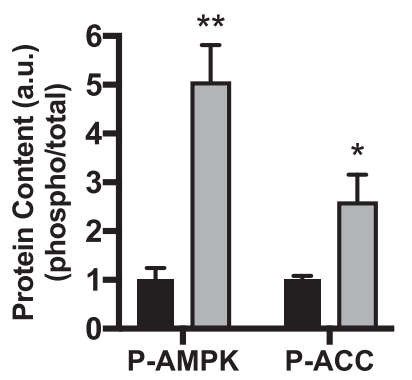

C

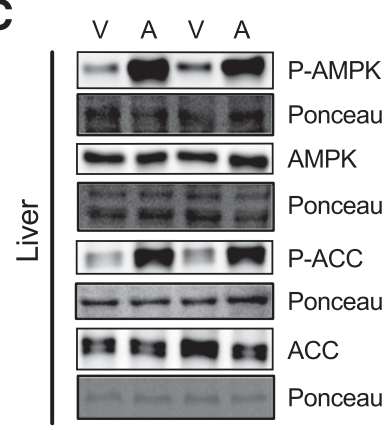

B

Triceps
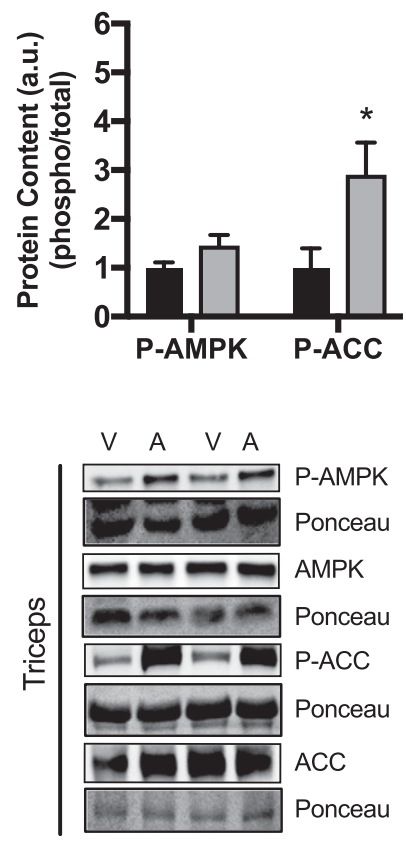

E

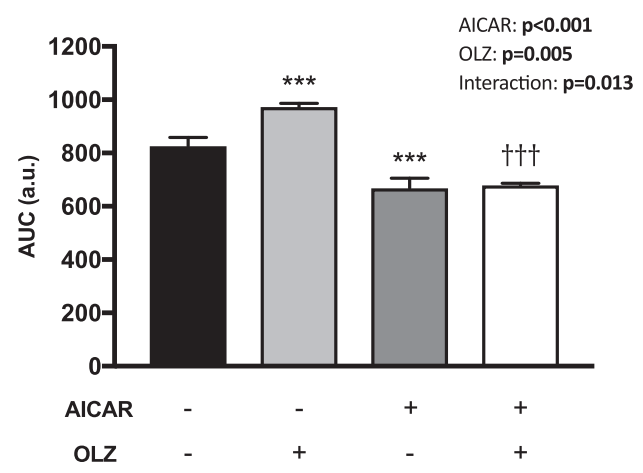

Fig. 1. AICAR protects against OLZinduced increases in blood glucose. Mice were injected intraperitoneally with either AICAR $(250 \mathrm{mg} / \mathrm{kg})$ or vehicle (saline), followed by tissue collection $\sim 20$ minutes postinjection. Phosphorylated and total AMPK and ACC protein content were measured using Western blotting. P-AMPK (T172) and P-ACC (S79) content in (A) liver and (B) triceps are expressed relative to total AMPK and ACC, respectively, and each was corrected for protein loading by normalizing to Ponceau S. (C) Representative Western blots for the quantified data in (A and B) are presented. A separate cohort of mice was injected intraperitoneally with one of four drug combinations (AICAR: $250 \mathrm{mg} / \mathrm{kg}$; OLZ: $5 \mathrm{mg} / \mathrm{kg}$ ), and blood glucose was measured before and 15, 30, 60 , and 90 minutes after drug treatment as shown in (D). (E) AUC of (D) was calculated. Data are presented as mean \pm S.E.M. ( $n=4-5$ /group). Two-way ANOVA main effects and interaction between treatment groups are shown on the right of $(\mathrm{E}) . \mathrm{V}=$ vehicle; $\mathrm{A}=$ AICAR. $* P<$ $0.05 ; * * P<0.01 ; * * * P<0.001$ relative to vehicle-treated group; $\dagger \dagger P<0.001$ relative to OLZ-treated group. $\mathrm{V}$, vehicle; A, AICAR.
OLZ Reduces RER, Oxygen Consumption, Activity Levels, and Heat Production. One of the side effects of OLZ that may contribute to increased blood glucose is upregulated fat oxidation (Klingerman et al., 2014). To assess this possibility and to determine whether AICAR can alter or improve these and other metabolic parameters such as activity and heat production, mice were housed in CLAMS metabolic cages and were given one of the four drug treatments. Within 4 hours, OLZ-treated mice, regardless of AICAR cotreatment, had a reduced RER, indicating increased

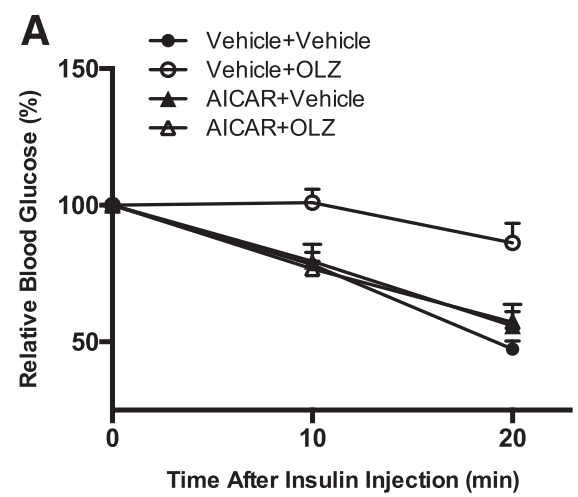

Time After Insulin Injection (min)

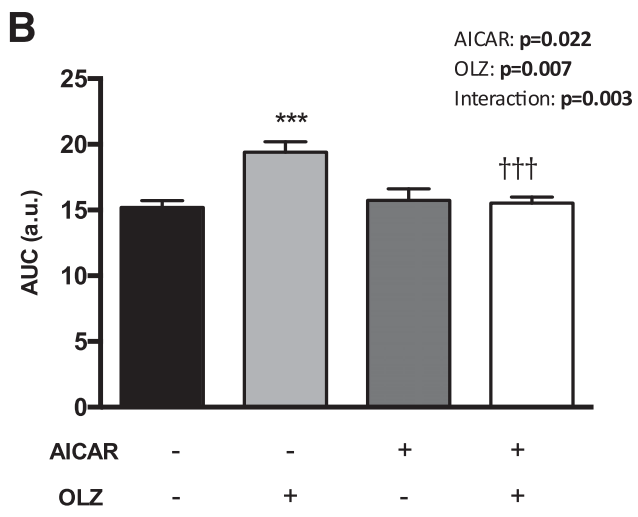

Fig. 2. AICAR protects against OLZ-induced reductions in the insulin response. Mice were injected intraperitoneally with one of four drug combinations (AICAR: $250 \mathrm{mg} / \mathrm{kg}$; OLZ: $5 \mathrm{mg} / \mathrm{kg}$ ), followed by a bolus injection of insulin (i.p., $0.75 \mathrm{U} / \mathrm{kg}$ ) 30 minutes after treatment. (A) Relative changes in blood glucose over time were plotted, and (B) AUC was calculated. Data are presented as mean \pm S.E.M. ( $n=6-8 /$ group). Two-way ANOVA main effects and interaction between treatment groups are shown on the right of (B). $* * * P<0.001$ relative to vehicle-treated group; $\dagger \dagger \uparrow P<0.001$ relative to OLZ-treated group. 


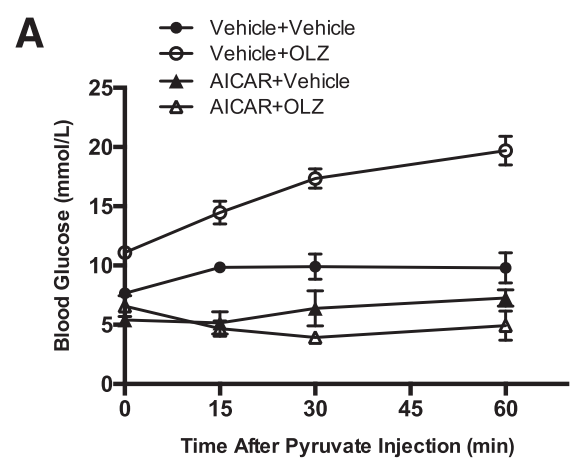

C

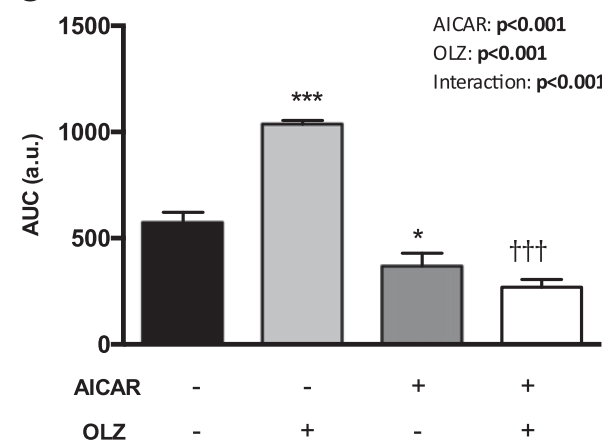

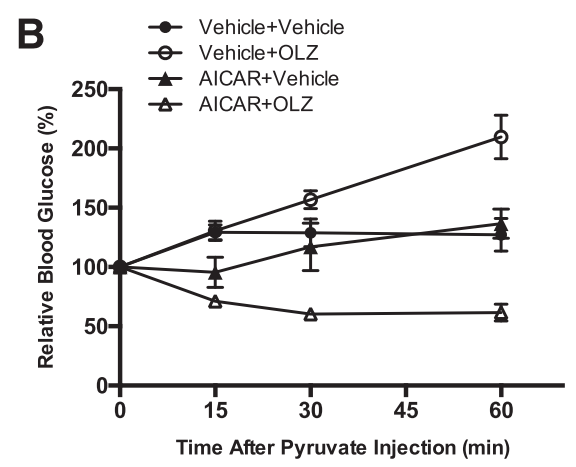

D

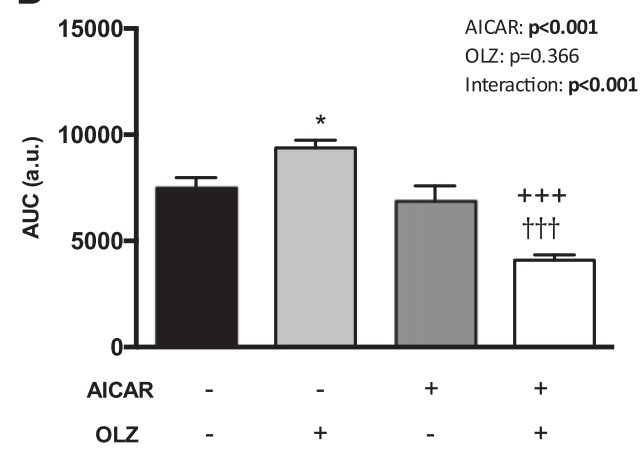

G

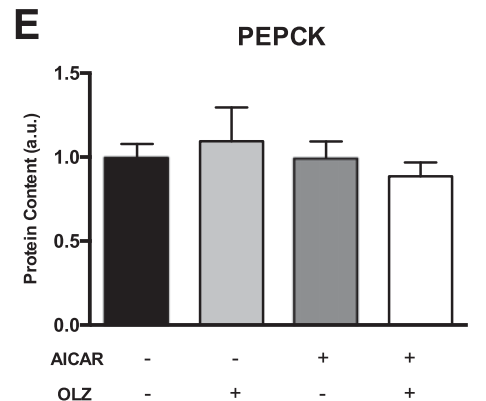

G6Pase

$\mathbf{F}$

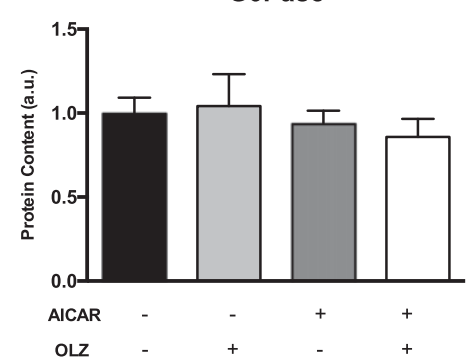

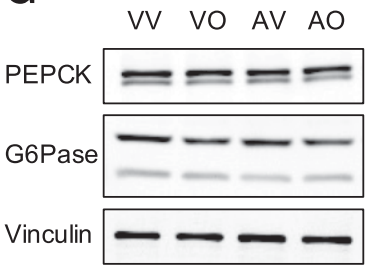

Fig. 3. AICAR attenuates pyruvate-induced excursions in blood glucose after OLZ treatment. Mice were injected intraperitoneally with one of four drug combinations (AICAR: $250 \mathrm{mg} / \mathrm{kg}$; OLZ: $5 \mathrm{mg} / \mathrm{kg}$ ) followed by a pyruvate injection (i.p., $2 \mathrm{~g} / \mathrm{kg}$ ) $30 \mathrm{minutes}$ after treatment. Blood glucose levels after pyruvate injection were measured and expressed as (A) absolute and (B) relative values, and the AUCs (C and D) were calculated. In a separate experiment, mice were injected intraperitoneally with one of four drug combinations (AICAR: $250 \mathrm{mg} / \mathrm{kg}$; OLZ: $5 \mathrm{mg} / \mathrm{kg}$ ), followed by tissue collection $\sim 20$ minutes post-treatment. Liver PEPCK and G6Pase protein content were analyzed using Western blotting. Quantified protein content of (E) PEPCK and (F) G6Pase are shown, both expressed relative to vinculin loading control (G6Pase and PEPCK were run on the same gel). (G) Representative Western blots for the quantified data in ( $\mathrm{E}$ and F) are presented. Data are presented as mean \pm S.E.M. $(n=4-7 /$ group $)$. Two-way ANOVA main effects and interactions between treatment groups are shown on the right of $(\mathrm{C}$ and $\mathrm{D}) . * P<0.05$; $* * * P<0.001$ relative to vehicle-treated group; $\dagger \dagger \dagger P<0.001$ relative to OLZ-treated group; $+++P<0.001$ relative to AICAR-treated group.

reliance on fat oxidation $(P<0.001)$ (Fig. 4 , A and B). Both OLZ-treated groups demonstrated reduced oxygen consumption regardless of AICAR treatment $\left(\mathrm{VO}_{2}\right)(P<0.01)$ (Fig. 4, C and $\mathrm{D})$. Although their activity is already reduced during the light cycle, both OLZ-treated groups, regardless of AICAR treatment, showed significantly reduced activity levels $(P<$ 0.001) (Fig. 4, E and F). Similarly, heat production was reduced in both OLZ-treated groups $(P<0.01)$ (Fig. 4G).

Peripheral AICAR Treatment Activates Hypothalamic AMPK Signaling. Previous work has demonstrated that the intracerebroventricular administration of either OLZ or AICAR activates AMPK signaling in the hypothalamus, and this was associated with increases in markers of hepatic glucose production (Ikegami et al., 2013). Given this finding, we were interested in determining whether the peripheral administration of AICAR, while protecting against OLZinduced hyperglycemia, also activates hypothalamic AMPK signaling. Blood glucose was measured to confirm that each treatment elicited the expected response. At 20 minutes' posttreatment, there was a main effect of $\operatorname{AICAR}(P<0.001)$ and OLZ $(P=0.003)$ treatment on blood glucose (Fig. 5A). No significant differences were found in the hypothalamic P-AMPK/AMPK ratio between groups; however, there was a main effect of AICAR on the hypothalamic P-ACC/ACC ratio $(P=0.033$ ), indicating activation of AMPK (Herzig and Shaw, 2018) (Fig. 5, B and C).

Ability of AICAR to Protect against OLZ-Induced Hyperglycemia, Reduced Insulin Response, and Pyruvate Intolerance is Intact in HFD-Induced Obese Mice. Since the acute effects of OLZ are exacerbated in 

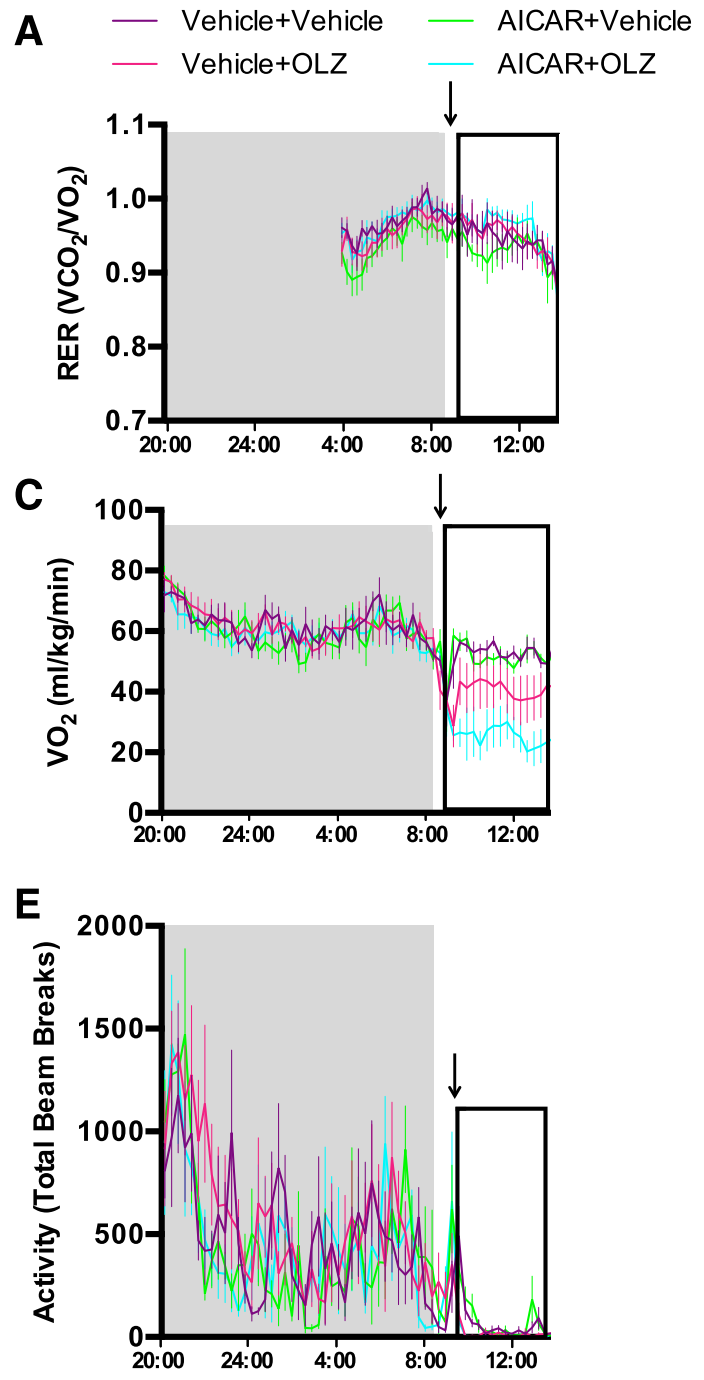

G

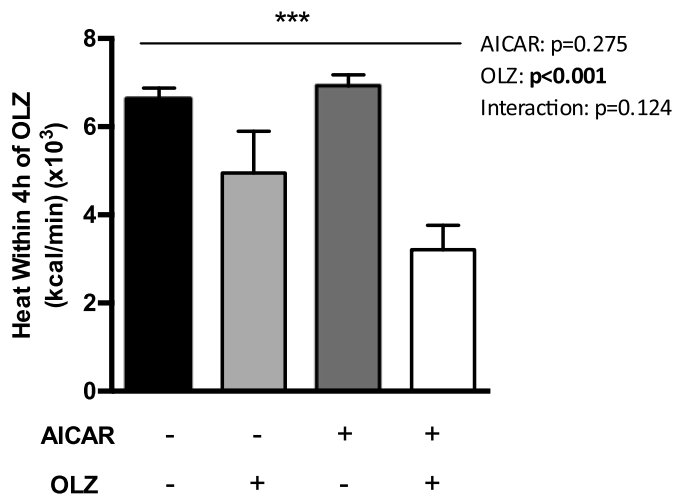

B

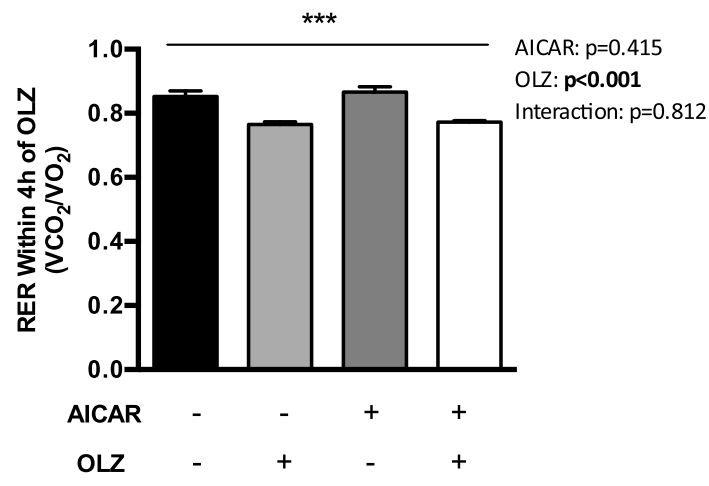

D

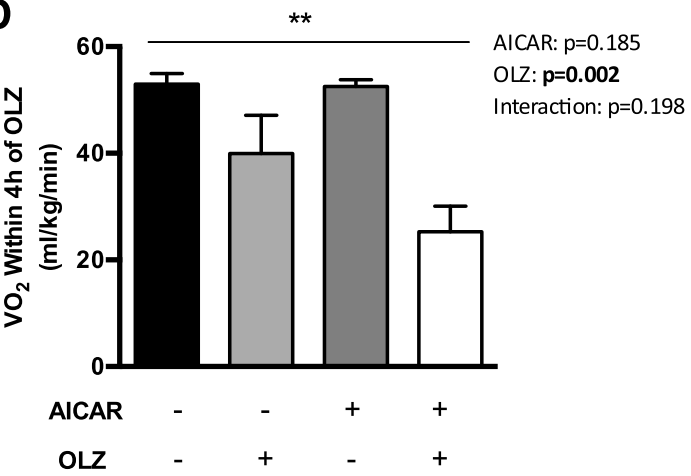

$\mathbf{F}$

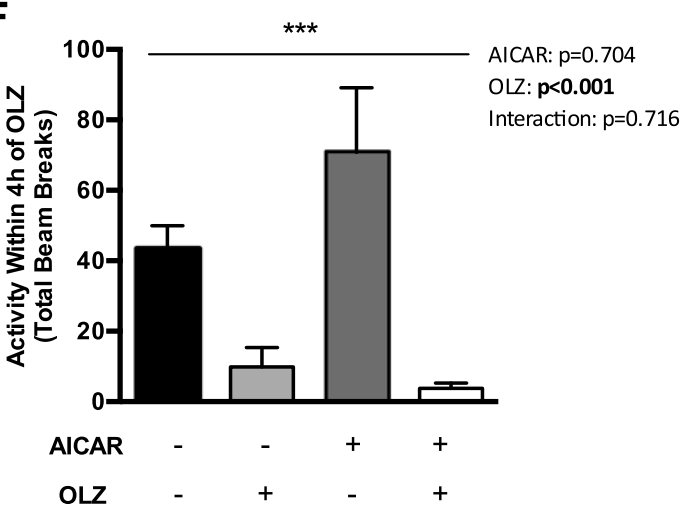

Fig. 4. OLZ reduces RER, activity levels, oxygen consumption, and heat production. Mice were acclimated in individually housed CLAMS units. Mice were injected with one of four drug combinations (AICAR: $250 \mathrm{mg} / \mathrm{kg}$; OLZ: $5 \mathrm{mg} / \mathrm{kg}$ ) at 9:30 AM (indicated by arrow) and then immediately put back into the CLAMS for 24 hours. (A) RER over dark and light cycles and (B) average RER during the first 4 hours after drug treatment. (C) VO ${ }_{2}$ over dark and light cycles and (D) average $\mathrm{VO}_{2}$ during the first 4 hours after drug treatment. (E) Activity over dark and light cycles and (F) average activity during the first 4 hours after drug treatment. (G) Average heat production during the first 4 hours after drug treatment. The shaded area represents the dark cycle, and a black rectangle indicates the 4-hour period after drug treatment in (A, C, and E). Data are presented as mean \pm S.E.M. ( $n=5-6 /$ group). Twoway ANOVA main effects and interactions between treatment groups are shown on the right of (B, D, F, and G), and as a line above each graph. Main effect of OLZ: $* * P<0.01 ; * * * P<0.001$

conditions of preexisting obesity and impaired glucose tolerance (Townsend et al., 2018), we wanted to determine whether the protective effects of AICAR were maintained in obese mice that had been fed a HFD. Mice were fed a HFD containing $60 \%$ of kilocalories from fat. Although the macronutrient content of this diet is likely not representative of what is 
A

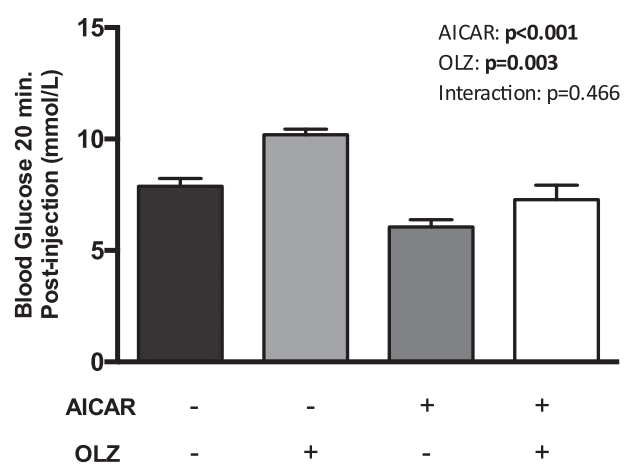

C

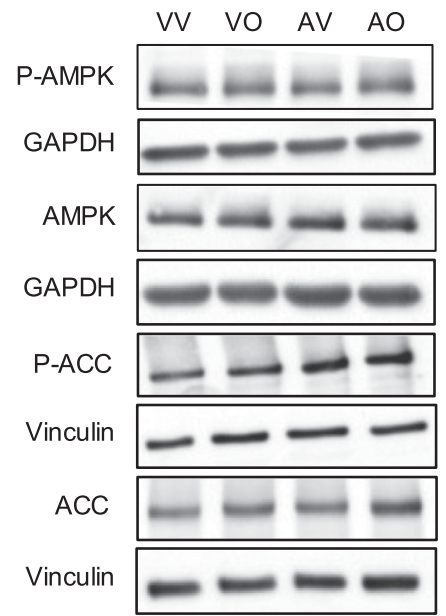

B Vehicle+Vehicle $\square$ AICAR+Vehicle $\square$ Vehicle+OLZ $\square$ AICAR+OLZ

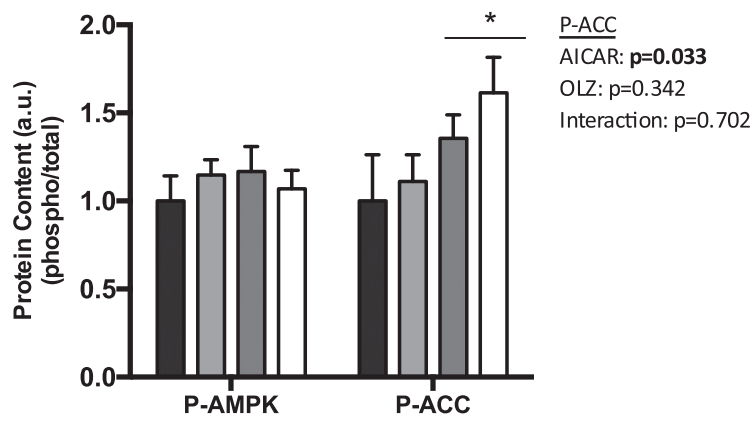

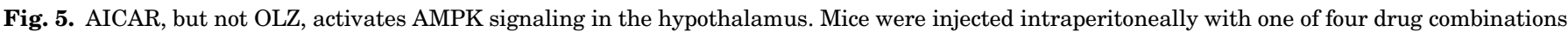

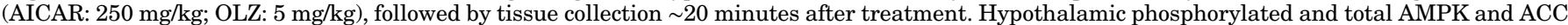

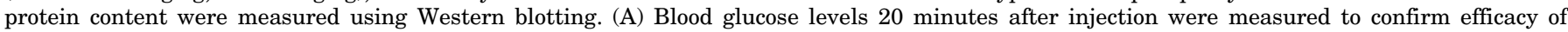

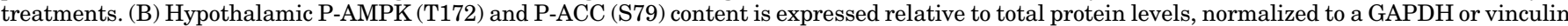

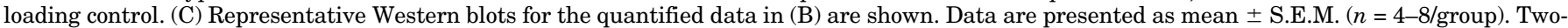

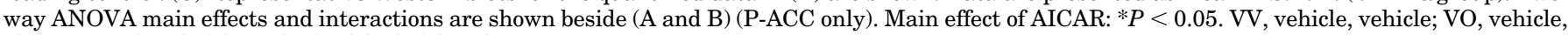
olanzapine; AV, AICAR vehicle; AO, AICAR olanzapine.

consumed by individuals with obesity and insulin resistance, we used it as a model to rapidly induce metabolic dysfunction. The average weight of the mice after 28 days of a HFD was $36.7 \pm 1 \mathrm{~g}$. OLZ significantly increased blood glucose levels compared with those in vehicle-treated mice $(P<0.001)$, and this effect was completely prevented by cotreatment with AICAR $(P<0.001)$ (Fig. 6, A and B). To investigate whether the protective effect of AICAR in terms of the insulin response is still intact in obese mice, an ITT was performed after pretreatment with each of the four drug combinations. Calculating the AUC up to 20 minutes after insulin treatment did not result in a significant increase with OLZ treatment; however, if the AUC calculation was extended to 30 minutes post-insulin, OLZ-treated obese mice demonstrated a blunted insulin response $(P<0.05)$, and this was alleviated by AICAR cotreatment $(P<0.001)$ (Fig. 6, C and D). To examine whether AICAR can inhibit pyruvate-induced excursions in blood glucose in obese mice, a pyruvate tolerance test was performed after pretreatment with each of the four drug combinations. Blood glucose levels after pyruvate injection were drastically increased with OLZ pretreatment $(P<0.001)$, and this was significantly blunted by $\operatorname{AICAR}(P<0.001)$ (Fig. 6, E and F).

\section{Discussion}

Long-term SGA use leads to weight gain and increases the risk of developing cardiometabolic diseases (Ceriello et al., 2008; Domecq et al., 2015). In addition, more recent work has shown that SGAs such as olanzapine can rapidly and directly induce marked impairments in glucose homeostasis (Houseknecht et al., 2007; Chintoh et al., 2008). Unfortunately, many commonly prescribed glucose-lowering medications are only partially effective in reducing these changes in blood glucose (Boyda et al., 2012). In the current study, we demonstrate that AICAR increases hepatic and skeletal muscle AMPK activity as demonstrated by increases in the phosphorylation of AMPK or its downstream substrate, ACC. The phosphorylation of ACC has been reported to be a more sensitive marker of AMPK activity than AMPK phosphorylation as it considers allosteric activation of AMPK (Gowans et al., 2013). The dissimilar increases in AMPK/ACC phosphorylation in liver and muscle likely speak to tissue-specific differences in the responsiveness to AICAR. Cotreatment with AICAR completely protected against OLZ-induced impairments in glucose homeostasis in both lean and obese mice. 


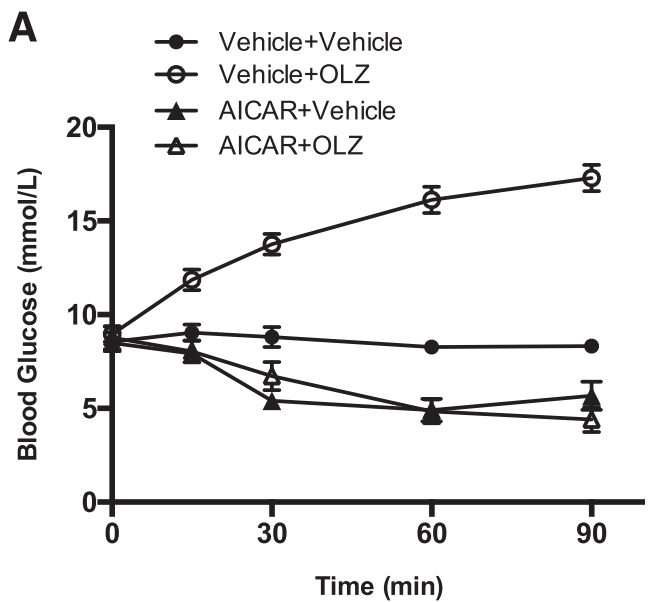

B
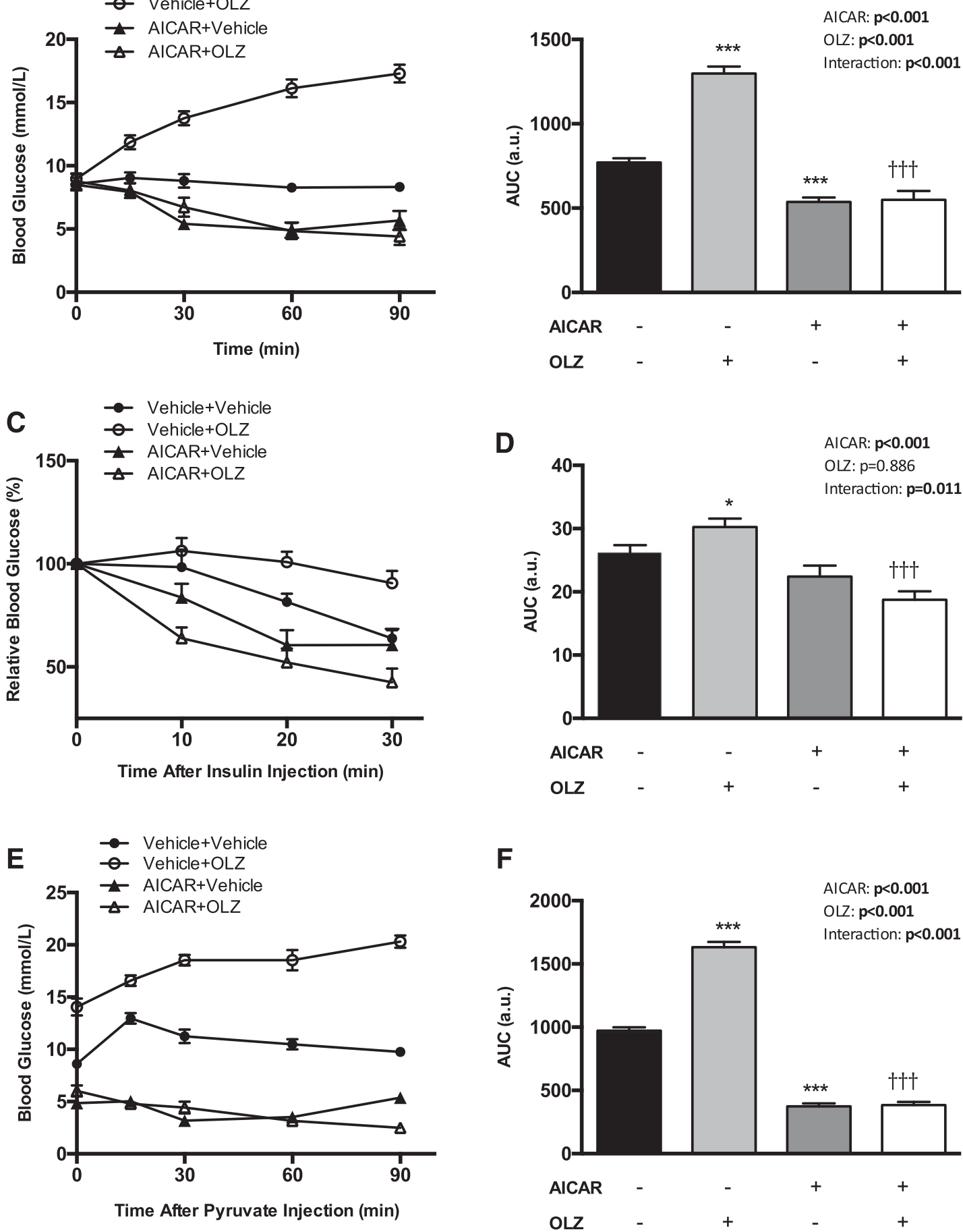

Fig. 6. AICAR protects against OLZ-induced hyperglycemia, reduced insulin response, and pyruvate intolerance in HFD-induced obese mice. Mice were fed a HFD for 28 days. After this period, mice were injected intraperitoneally with one of four drug combinations (AICAR: $250 \mathrm{mg} / \mathrm{kg}$; OLZ: $5 \mathrm{mg} / \mathrm{kg}$ ), and blood glucose was measured prior to and 15, 30, 60, and 90 minutes after drug treatment as shown in (A). (B) AUC of (A) was calculated. A separate cohort of obese mice were injected intraperitoneally with one of four drug combinations, followed by a bolus injection of insulin (i.p., $0.75 \mathrm{U} / \mathrm{kg}$ ) $30 \mathrm{minutes}$ after treatment. (C) Relative changes in blood glucose over time were plotted, and (D) AUC was calculated. (E) Blood glucose levels after pyruvate injection, and (F) AUC of (E). Data are presented as mean \pm S.E.M. $(n=7 /$ group). Two-way ANOVA main effects and interaction between treatment groups are shown on the right of (B, D, and F). *P<0.05; ***P $<0.001$ relative to vehicle-treated group; $\uparrow \dagger \uparrow P<0.001$ relative to OLZ-treated group.

Previous studies have shown that OLZ induces insulin resistance in mice (Castellani et al., 2017; Townsend et al., 2018 ) and that AICAR improves whole-body insulin action in insulin-resistant high-fat diet-fed rats (Iglesias et al., 2002).
These effects of AICAR were attributed to improvements in skeletal muscle glucose uptake (Iglesias et al., 2002). In the present study, AICAR protected against OLZ-induced impairments in the whole-body insulin response as measured using an 
ITT, which could relate to clinical insulin resistance, although tissue-specific effects on insulin signaling would likely need to be incorporated. Reductions in the insulin response, although prevented by AICAR, may not be a causal event in OLZ-induced hyperglycemia. In support of this premise, we have recently shown that glucagon receptor knockout mice are protected against OLZ-induced hyperglycemia despite severe insulin resistance (Castellani et al., 2017). These mice showed reductions in hepatic glucose output secondary to decreases in the protein content of the gluconeogenic enzymes PEPCK and G6Pase, suggesting an important role for the liver in OLZinduced hyperglycemia (Castellani et al., 2017).

With regard to liver glucose output, we demonstrate that peripheral injection of OLZ potentiates the blood glucose response to a pyruvate challenge. These findings, consistent with previous work using hyperinsulinemic, euglycemic clamps (Houseknecht et al., 2007; Chintoh et al., 2008), indicate that OLZ increases hepatic glucose production. Similar to the protective effect against OLZ-induced reductions in the insulin response, cotreatment with AICAR completely protected against OLZ-induced excursions in blood glucose after a pyruvate challenge; however, the mechanisms mediating this effect are not clear. Although AICAR activates AMPK and the prolonged activation of this enzyme reduces hepatic gluconeogenic capacity (Foretz et al., 2005), it has recently been shown that AICAR-mediated inhibition of glucose production was intact in liver-specific AMPK knockout mice (Hasenour et al., 2014). AICAR, in addition to increasing ZMP, has also been reported to increase AMP secondary to an inhibition of mitochondrial respiration (Hasenour et al., 2014). As both AMP (Underwood and Newsholme, 1965) and ZMP (Vincent et al., 1991) can directly inhibit gluconeogenic enzymes, this could be a potential mechanism through which AICAR could be blunting OLZ-induced increases in liver glucose production.

In contrast to the current investigation, recent studies have demonstrated that intracerebroventricular administration of OLZ is associated with increased hepatic glucose production in an AMPK-dependent manner, which was mirrored by increases in liver PEPCK and G6Pase mRNA expression (Martins et al., 2010; Ikegami et al., 2013). These studies showed that the effects of central OLZ administration are dependent on AMPK activation in the hypothalamus (Martins et al., 2010; Ikegami et al., 2013). In the current study, peripheral injection of AICAR, but not OLZ, activated AMPK signaling in the hypothalamus. These findings provide evidence that peripheral OLZ treatment, an approach more clinically relevant than intracerebroventricular administration, does not induce hyperglycemia through increasing AMPK activity in the hypothalamus. Our findings further suggest that the peripheral (liver, muscle, etc.) effects of AICAR are sufficient to overcome any hyperglycemic effects of increased hypothalamic AMPK activation. Furthermore, since AICAR is unable to effectively cross the blood-brain barrier (Marangos et al., 1990), this increase in hypothalamic AMPK activity may represent a compensatory response to AICAR-induced reductions in blood glucose.

OLZ displays potent sedative effects (Albaugh et al., 2011), and here we extend this to show that OLZ significantly reduces overall activity levels, with or without the presence of AICAR. OLZ is reported to increase reliance on fat oxidation (i.e., it reduces $\mathrm{RER}$ ), which can lead to plasma glucose accumulation (Klingerman et al., 2014, 2015), thought to be due to central effects of OLZ (Klingerman et al., 2015). Similarly, we show that OLZ, regardless of AICAR cotreatment, reduces $R E R$, indicating increases in fat oxidation. Notably, since AICAR did not alter the RER, changes in substrate metabolism may be ruled out as a potential mechanism through which AICAR may be exerting its antihyperglycemic effects.

Since the acute effects of OLZ are exacerbated in conditions of preexisting obesity and impaired glucose homeostasis (Townsend et al., 2018), and before taking antipsychotics, individuals with schizophrenia are often overweight and/or display perturbations in glucose homeostasis (Subramaniam et al., 2014), the ability of AICAR to protect against OLZinduced disturbances in glucose homeostasis were further examined in HFD-induced obese mice. Similar to lean mice, in obese mice, AICAR prevented the OLZ-induced rise in blood glucose, acute impairments in the insulin response, and increases in markers of hepatic glucose output after a pyruvate challenge. Based on previous research (Subramaniam et al., 2014), the ability of AICAR to protect against OLZ-induced metabolic disturbances in the presence of preexisting obesity, glucose intolerance, and insulin resistance would appear to be more clinically relevant in terms of schizophrenic individuals taking OLZ.

Overall, the current report provides evidence that cotreatment with AICAR completely protects against OLZ-induced impairments in glucose homeostasis in both lean and obese mice. This result is likely mediated through improvements in insulin responsiveness and reductions in hepatic glucose output. The results of this study highlight the ability of AICAR to mitigate the undesirable acute effects of OLZ. Furthermore, the current results demonstrate that agents targeting both hepatic gluconeogenic capacity and skeletal muscle glucose homeostasis may be a potential avenue through which to prevent SGA-induced impairments in glucose homeostasis and insulin action. Further studies investigating the ability of AICAR to mitigate the effects of chronic OLZ treatment would be beneficial to provide more clinically relevant data regarding long-term OLZ use.

\section{Authorship Contributions}

Participated in research design: Bush, Townsend, Wright.

Conducted experiments: Bush, Townsend.

Performed data analysis: Bush, Wright.

Wrote or contributed to the writing of the manuscript: Bush, Wright.

\section{References}

Albaugh VL, Judson JG, She P, Lang CH, Maresca KP, Joyal JL, and Lynch CJ (2011) Olanzapine promotes fat accumulation in male rats by decreasing physical activity, repartitioning energy and increasing adipose tissue lipogenesis while impairing lipolysis. Mol Psychiatry 16:569-581.

Bergman RN and Ader M (2005) Atypical antipsychotics and glucose homeostasis. $J$ Clin Psychiatry 66:504-514.

Boyda HN, Procyshyn RM, Tse L, Hawkes E, Jin CH, Pang CC, Honer WG, and Barr AM (2012) Differential effects of 3 classes of antidiabetic drugs on olanzapineinduced glucose dysregulation and insulin resistance in female rats. $J$ Psychiatry Neurosci 37:407-415.

Boyda HN, Tse L, Procyshyn RM, Wong D, Wu TK, Pang CC, and Barr AM (2010) A parametric study of the acute effects of antipsychotic drugs on glucose sensitivity in an animal model. Prog Neuropsychopharmacol Biol Psychiatry 34:945-954.

Bun S, Yonemori K, Akagi T, Noguchi E, Shimoi T, Shimomura A, Yunokawa M, Shimizu C, Fujiwara Y, Makino Y, et al. (2017) Feasibility of olanzapine, multi acting receptor targeted antipsychotic agent, for the prevention of emesis caused by continuous cisplatin- or ifosfamide-based chemotherapy. Invest New Drugs 36: 151-155.

Castellani LN, Peppler WT, Miotto PM, Bush N, and Wright DC (2018) Exercise protects against olanzapine-induced hyperglycaemia in male C57BL6/J mice. Sci Rep 8:772. 
Castellani LN, Peppler WT, Sutton CD, Whitfield J, Charron MJ, and Wright DC (2017) Glucagon receptor knockout mice are protected against acute olanzapineinduced hyperglycemia. Psychoneuroendocrinology 82:38-45.

Ceriello A, Esposito K, Piconi L, Ihnat MA, Thorpe JE, Testa R, Boemi M, and Giugliano D (2008) Oscillating glucose is more deleterious to endothelia function and oxidative stress than mean glucose in normal and type 2 diabetic patients. Diabetes 57:1349-1354.

Chelkeba L, Gidey K, Mamo A, Yohannes B, Matso T, and Melaku T (2017) Olanzapine for chemotherapy-induced nausea and vomiting: systematic review and meta-analysis. Pharm Pract (Granada) 15:877.

Chintoh AF, Mann SW, Lam L, Giacca A, Fletcher P, Nobrega J, and Remington G (2009) Insulin resistance and secretion in vivo: effects of different antipsychotics in an animal model. Schizophr Res 108:127-133.

Chintoh AF, Mann SW, Lam L, Lam C, Cohn TA, Fletcher PJ, Nobrega JN, Giacca A and Remington G (2008) Insulin resistance and decreased glucose-stimulated insulin secretion after acute olanzapine administration. J Clin Psychopharmacol 28 494-499.

Devlin AM and Panagiotopoulos C (2015) Metabolic side effects and pharmacogenetics of second-generation antipsychotics in children. Pharmacogenomics 16: 981-996.

Domecq JP, Prutsky G, Leppin A, Sonbol MB, Altayar O, Undavalli C, Wang Z, Elraiyah T, Brito JP, Mauck KF, et al. (2015) Clinical review: drugs commonly associated with weight change: a systematic review and meta-analysis. $J$ Clin Endocrinol Metab 100:363-370.

Firth J, Cotter J, Elliott R, French P, and Yung AR (2015) A systematic review and meta-analysis of exercise interventions in schizophrenia patients. Psychol Med $\mathbf{4 5}$ 1343-1361.

Foretz M, Ancellin N, Andreelli F, Saintillan Y, Grondin P, Kahn A, Thorens B, Vaulont S, and Viollet B (2005) Short-term overexpression of a constitutively active form of AMP-activated protein kinase in the liver leads to mild hypoglycemia and fatty liver. Diabetes 54:1331-1339.

Gaidhu MP, Fediuc S, Anthony NM, So M, Mirpourian M, Perry RL, and Ceddia RB (2009) Prolonged AICAR-induced AMP-kinase activation promotes energy dissipation in white adipocytes: novel mechanisms integrating HSL and ATGL. J Lipid Res 50:704-715.

Goodyear LJ (2008) The exercise pill-too good to be true? N Engl J Med 359: $1842-1844$

Gowans GJ, Hawley SA, Ross FA, and Hardie DG (2013) AMP is a true physiological regulator of AMP-activated protein kinase by both allosteric activation and enhancing net phosphorylation. Cell Metab 18:556-566.

Guerrieri D and van Praag H (2015) Exercise-mimetic AICAR transiently benefits brain function. Oncotarget 6:18293-18313.

Guigas B, Taleux N, Foretz M, Detaille D, Andreelli F, Viollet B, and Hue L (2007) AMP-activated protein kinase-independent inhibition of hepatic mitochondrial oxidative phosphorylation by AICA riboside. Biochem J 404:499-507.

Hahn MK, Wolever TM, Arenovich T, Teo C, Giacca A, Powell V, Clarke L, Fletcher P, Cohn T, McIntyre RS, et al. (2013) Acute effects of single-dose olanzapine on metabolic, endocrine, and inflammatory markers in healthy controls. J Clin Psychopharmacol 33:740-746.

Hasenour CM, Ridley DE, Hughey CC, James FD, Donahue EP, Shearer J, Viollet B Foretz M, and Wasserman DH (2014) 5-Aminoimidazole-4-carboxamide-1- $\beta$-D ribofuranoside (AICAR) effect on glucose production, but not energy metabolism, is independent of hepatic AMPK in vivo. J Biol Chem 289:5950-5959.

Herzig S and Shaw RJ (2018) AMPK: guardian of metabolism and mitochondrial homeostasis. Nat Rev Mol Cell Biol 19:121-135.

Holloszy JO (2003) A forty-year memoir of research on the regulation of glucose transport into muscle. Am J Physiol Endocrinol Metab 284:E453-E467.

Houseknecht KL, Robertson AS, Zavadoski W, Gibbs EM, Johnson DE, and Rollema H (2007) Acute effects of atypical antipsychotics on whole-body insulin resistance in rats: implications for adverse metabolic effects. Neuropsychopharmacology 32 289-297.

Iglesias MA, Ye JM, Frangioudakis G, Saha AK, Tomas E, Ruderman NB, Cooney GJ, and Kraegen EW (2002) AICAR administration causes an apparent enhancement of muscle and liver insulin action in insulin-resistant high-fat-fed rats. $D i$ abetes 51:2886-2894.

Ikegami M, Ikeda H, Ohashi T, Ohsawa M, Ishikawa Y, Kai M, Kamei A, and Kamei J (2013) Olanzapine increases hepatic glucose production through the activation of hypothalamic adenosine $55^{\prime}$-monophosphate-activated protein kinase. Diabetes Obes Metab 15:1128-1135.

Jørgensen SB, Viollet B, Andreelli F, Frøsig C, Birk JB, Schjerling P, Vaulont S, Richter EA, and Wojtaszewski JF (2004) Knockout of the alpha2 but not alpha1 $5^{\prime}$ AMP-activated protein kinase isoform abolishes 5-aminoimidazole-4-carboxamide1-beta-4-ribofuranosidebut not contraction-induced glucose uptake in skeletal muscle. J Biol Chem 279:1070-1079.

Kapur S, VanderSpek SC, Brownlee BA, and Nobrega JN (2003) Antipsychotic dosing in preclinical models is often unrepresentative of the clinical condition: a suggested solution based on in vivo occupancy. J Pharmacol Exp Ther 305:625-631.
Kessing LV, Thomsen AF, Mogensen UB, and Andersen PK (2010) Treatment with antipsychotics and the risk of diabetes in clinical practice. $B r J$ Psychiatry 197:266-271.

Kjøbsted R, Treebak JT, Fentz J, Lantier L, Viollet B, Birk JB, Schjerling P, Björnholm M, Zierath JR, and Wojtaszewski JF (2015) Prior AICAR stimulation increases insulin sensitivity in mouse skeletal muscle in an AMPK-dependent manner. Diabetes 64:2042-2055.

Klingerman CM, Stipanovic ME, Bader M, and Lynch CJ (2014) Second-generation antipsychotics cause a rapid switch to fat oxidation that is required for survival in C57BL/6J mice. Schizophr Bull 40:327-340.

Klingerman CM, Stipanovic ME, Hajnal A, and Lynch CJ (2015) Acute metabolic effects of olanzapine depend on dose and injection site. Dose Response 13: 1559325815618915

Lord CC, Wyler SC, Wan R, Castorena CM, Ahmed N, Mathew D, Lee S, Liu C, and Elmquist JK (2017) The atypical antipsychotic olanzapine causes weight gain by targeting serotonin receptor $2 \mathrm{C}$. J Clin Invest 127:3402-3406.

MacPherson RE, Baumeister P, Peppler WT, Wright DC, and Little JP (2015) Reduced cortical BACE1 content with one bout of exercise is accompanied by declines in AMPK, Akt, and MAPK signaling in obese, glucose-intolerant mice. J Appl Physiol (1985) 119:1097-1104.

Maher AR, Maglione M, Bagley S, Suttorp M, Hu JH, Ewing B, Wang Z, Timmer M, Sultzer D, and Shekelle PG (2011) Efficacy and comparative effectiveness of atypical antipsychotic medications for off-label uses in adults: a systematic review and meta-analysis. JAMA 306:1359-1369.

Marangos PJ, Loftus T, Wiesner J, Lowe T, Rossi E, Browne CE, and Gruber HE (1990) Adenosinergic modulation of homocysteine-induced seizures in mice. Epilepsia 31:239-246.

Martins PJ, Haas M, and Obici S (2010) Central nervous system delivery of the antipsychotic olanzapine induces hepatic insulin resistance. Diabetes 59:2418-2425.

Monaco C, Whitfield J, Jain SS, Spriet LL, Bonen A, and Holloway GP (2015) Activation of $\mathrm{AMPK} \alpha 2$ is not required for mitochondrial FAT/CD36 accumulation during exercise. PLoS One 10:e126122.

Narkar VA, Downes M, Yu RT, Embler E, Wang YX, Banayo E, Mihaylova MM, Nelson MC, Zou Y, Juguilon H, et al. (2008) AMPK and PPARdelta agonists are exercise mimetics. Cell 134:405-415.

Newcomer JW (2005) Second-generation (atypical) antipsychotics and metabolic effects: a comprehensive literature review. CNS Drugs 19 (Suppl 1):1-93.

O'Neill HM, Lally JS, Galic S, Thomas M, Azizi PD, Fullerton MD, Smith BK, Pulinilkunnil T, Chen Z, Samaan MC, et al. (2014) AMPK phosphorylation of ACC2 is required for skeletal muscle fatty acid oxidation and insulin sensitivity in mice. Diabetologia 57:1693-1702.

Pillinger T, Beck K, Gobjila C, Donocik JG, Jauhar S, and Howes OD (2017) Impaired glucose homeostasis in first-episode schizophrenia: a systematic review and metaanalysis. JAMA Psychiatry 74:261-269.

Pringsheim T and Gardner DM (2014) Dispensed prescriptions for quetiapine and other second-generation antipsychotics in Canada from 2005 to 2012: a descriptive study. CMAJ Open 2:E225-E232.

Reynolds GP and McGowan OO (2017) Mechanisms underlying metabolic disturbances associated with psychosis and antipsychotic drug treatment. $J$ Psychopharmacol 31:1430-1436.

Richter EA, Garetto LP, Goodman MN, and Ruderman NB (1982) Muscle glucose metabolism following exercise in the rat: increased sensitivity to insulin. $J$ Clin Invest 69:785-793.

Richter EA and Hargreaves M (2013) Exercise, GLUT4, and skeletal muscle glucose uptake. Physiol Rev 93:993-1017.

Smith PK, Krohn RI, Hermanson GT, Mallia AK, Gartner FH, Provenzano MD, Fujimoto EK, Goeke NM, Olson BJ, and Klenk DC (1985) Measurement of protein using bicinchoninic acid. Anal Biochem 150:76-85.

Subramaniam M, Lam M, Guo ME, He VY, Lee J, Verma S, and Chong SA (2014) Body mass index, obesity, and psychopathology in patients with schizophrenia. $J$ Clin Psychopharmacol 34:40-46.

Townsend LK, Peppler WT, Bush ND, and Wright DC (2018) Obesity exacerbates the acute metabolic side effects of olanzapine. Psychoneuroendocrinology 88:121-128. Underwood AH and Newsholme EA (1965) Some properties of fructose 1,6-diphosphatase of rat liver and their relation to the control of gluconeogenesis. Biochem $J$ 95:767-774 van Nimwegen LJ, Storosum JG, Blumer RM, Allick G, Venema HW, de Haan L, Becker H, van Amelsvoort T, Ackermans MT, Fliers E, et al. (2008) Hepatic insulin resistance in antipsychotic naive schizophrenic patients: stable isotope studies of glucose metabolism. J Clin Endocrinol Metab 93:572-577.

Vincent MF, Marangos PJ, Gruber HE, and Van den Berghe G (1991) Inhibition by AICA riboside of gluconeogenesis in isolated rat hepatocytes. Diabetes 40: 1259-1266.

Address correspondence to: David C. Wright, Room 334 Animal Sciences Building, Department of Human Health and Nutritional Sciences, University of Guelph, Guelph, Ontario N1G 2W1, Canada. E-mail: dcwright@uoguelph.ca 